Voix et Images

volxetimages

\title{
Le roman québécois sous le regard de l'autre
}

\section{Robert Major}

Volume 16, numéro 3 (48), printemps 1991

\section{François Charron}

URI : https://id.erudit.org/iderudit/200927ar

DOI : https://doi.org/10.7202/200927ar

Aller au sommaire du numéro

Éditeur(s)

Université du Québec à Montréal

ISSN

0318-9201 (imprimé)

1705-933X (numérique)

Découvrir la revue

Citer cet article

Major, R. (1991). Le roman québécois sous le regard de l'autre. Voix et Images, 16(3), 526-532. https://doi.org/10.7202/200927ar d'utilisation que vous pouvez consulter en ligne.

https://apropos.erudit.org/fr/usagers/politique-dutilisation/ 


\section{Essai}

\section{Le roman québécois sous le regard de l'autre}

\section{par Robert Major, Université d'Ottawa}

Il y a longtemps que le public cultivé canadien-anglais s'intéresse à la littérature québécoise et que des universitaires anglophones se penchent sur les œuvres québécoises. Sympathiques ou simplement curieux, ces anglophones signalent et scrutent ces œuvres, quelquefois les traduisent; ils y cherchent les indices d'une spécificité fascinante et peut-être aussi les signes avant-coureurs d'un destin enfin partagé, malgré qu'on en ait. La réciproque n'est pas vraie, on le sait. Le recensement établi par Philip Stratford en 1977 sur les traductions de livres canadiens d'une langue officielle à l'autre révélait un profond déséquilibre. La situation n'a guère changé depuis. Les traductions en français d'œuvres anglophones, ainsi que le signalait Sherry Simon plus récemment (Circuit, $\mathrm{n}^{\circ} 17$, juin 1987), ne font pas le poids face aux traductions made in Canada. Le milieu littéraire québécois est fermé aux œuvres canadiennes-anglaises et sans doute peut-on le regretter. Par nombrilisme, ou naïveté, ou indifférence, qui sait, les Québécois non seulement se privent de la connaissance d'œuvres remarquables mais, de plus, se maintiennent dans une ignorance dont ils feront éventuellement les frais. Tous ces traducteurs qui ahanent dans toutes les officines du fédéral à rendre en français des montagnes de paperasses bureaucratiques seraient peut-être mieux employés à traduire les œuvres de Robertson Davies, de Margaret Lawrence, de Farley Mowatt, de Pierre Berton et, pourquoi pas, de Ralph Connor.

Deux livres récents confirment cet intérêt séculaire des universitaires canadiens-anglais pour les écrivains québécois et contribuent à creuser davantage l'écart avec les universitaires québécois. Le retard de ces derniers à rendre la politesse est d'autant plus accusé.

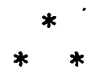

En vérité, le premier de ces livres, Moments postmodernes dans le roman québécois de Janet $M$. Paterson ${ }^{1}$, est autant sinon plus intéressé par les questions théoriques que par les œuvres 
québécoises. Plus, si on en juge par le graphisme de la page couverture qui met nettement en relief, par les caractères gras, les deux premiers mots du titre: Moments postmodernes. Mise en relief tout à fait justifiée et honnête. L'introduction et deux des sept chapitres de ce petit livre sont consacrés à l'explication de la poétique postmoderne. La réflexion théorique d'ailleurs ne se contente pas de ce statut de prolégomène: elle se déploie aussi amplement à l'intérieur des chapitres consacrés à l'analyse d'une œuvre particulière. Ainsi l'étude de Trou de mémoire (chapitre 3) contient une réflexion sur la portée idéologique de l'annotation critique, savante ou fictive; l'analyse de la Maison Trestler (chapitre 4) présente une longue parenthèse sur les problèmes débattus par les théoriciens de l'historiographie; le chapitre sur le Semestre (le cinquième) est consacré pour la moitié à un examen de la théorie de l'intertextualité; et celui sur D'Amour P.Q. (le septième) utilise plus de la moitié de ses pages à réfléchir, Barthes à l'appui, sur la représentation de l'écrivain dans le texte postmoderne.

Doit-on se plaindre de cette omniprésence de la théorie et du recours constant à l'exposé doctrinal qui réduisent quelquefois l'étude des romans à la portion congrue? Dans ces pages (Voix \& images, $n^{0} 42$, François Gallays déplorait récemment la grande confusion entourant l'utilisation des notions de modernisme et de postmodernisme. Or, voici justement un livre qui répond à ses attentes (et aux nôtres!), car il se propose de mettre de l'ordre dans les débats, d'expliquer clairement et succinctement les tenants et aboutissants d'une notion foncièrement vaseuse. Et, ma foi! force est de constater que Janet Paterson réussit. Son livre - toutes propositions gardées, étant donné le champ d'intérêt - est dénué de jargon, de tics à la mode et de prétention scientiste; mieux, il est précis, lisible et fort utile.

L'affaire n'était pas mince, si l'on songe que le propagandiste français du terme "postmoderne", Jean-François Lyotard, n'arrive pas à cerner sa signification dans un article qui se propose de le définir (ou se complaît à brouiller les pistes, ce qui revient au même) et que le mot véhicule une saveur apocalyptique avec des relents d'obscurantisme et de grande frayeur du millénaire qui n'incitent guère à la précision conceptuelle. Définition type: le postmodernisme [...] n'est pas le modernisme à sa fin, mais à l'état naissant, et cet état est récurrent (Lyotard)! Certes, le lecteur qui a quelques souvenirs de la signification des préfixes, ou tout simplement qui n'a pas la tête théorique ou pédantesque, sera excusé de ressentir une certaine perplexité ou une certaine lassitude devant ces confusions et ces débats doctrinaires de "lettresférits". Le mérite de ce livre est de feindre stratégiquement l'étonnement devant ces imprécisions agaçantes pour ensuite s'employer hardiment à examiner le sens et la pertinence du terme tant dans le domaine du roman que dans celui de la théorie littéraire (p. 10). 
La notion est donc examinée dans son histoire (anglo-américaine puis française), ses ambiguïtés, son champ sémantique et face à des termes contigus et concurrents. Ainsi, la notion est à distinguer de l'avant-garde dont elle n'a ni le cachet d'expérimentation radicale, ni les fortes tendances subversives, ni la violence, ni l'orientation politique; à distinguer également de la métafiction qui n'est qu'une caractéristique, une composante du postmoderne: le processus d'autoreprésentation dans le texte: Par ailleurs, le postmoderne ne saurait se confondre avec le nouveau roman, phénomène restreint à la France: quelques nouveaux romans d'ailleurs n'ont rien de l'esthétique postmoderne. Finalement, le postmoderne, paradoxalement, n'est pas une notion chronologique: il est inclus dans la modernité, ce dernier étant un terme vaste et englobant dont les frontières sont indéfinies.

Le postmoderne est une nouvelle poétique dont le fondement idéologique est la volonté de faire éclater les discours dominants et l'ordre des certitudes. Il est, en somme, une esthétique pour notre temps, incrédule, questionneur, sceptique et inquiet. Ainsi le roman postmoderne se caractérise par un certain nombre d'éléments précis. Le narrateur y est pluriel, diffus et contradictoire; sa vulnérabilité met en question la notion d'autorité et saborde toute volonté de totalisation. Le narrataire est lui aussi représenté et multiple et, forcément; ne peut adhérer auu discours du narrateur: d'abord parce que celui-ci n'offre pas de prises solides, ensuite parce que le narrataire est de toute façon sceptique. Au niveau de la diégèse, le roman postmoderne présente deux champs thématiques privilégiés: d'une part un discours inlassable sur l'écriture, la lecture, le travail critique, l'art et, d'autre part, une prédominance de tout ce qui peut indiquer la rupture, la pluralité, la fragmentation, l'ouverture indéfinie du sens. Finalement, au niveau du code, ce roman met constamment en relief la pratique du texte par l'autoreprésentation de l'écriture et par l'utilisation considérable de l'intertextualité dans tous ses aspects: allusions à profusion, intégration de fragments non littéraires, étalage d'érudition, croisement des genres (et conséquemment subversion générique), discours parodiques. Le lecteur est ainsi devant un roman qui exhibe toujours son propre fonctionnement par l'autoreprésentation, l'autoréflexivité, la mise en abyme.

S'agit-il d'exercices gratuits, narcissiques, témoins d'un épuisement de la littérature et d'une pulsion de mort dans la société ? Janet Paterson est bien consciente des menaces qui guettent le roman postmoderne: esthétisme, élitisme, procédé, schizophrénie textuelle, néo-maniérisme (sans compter la désaffection totale du public lecteur). Mais elle voit plutôt dans ces textes une force libératrice à l'œuvre. Cette contestation des discours d'autorité (et au premier titre de celui du narrateur qui pourrait prétendre ordonner le récit), ce comportement essentiellement iconoclaste à l'égard de la tradition 
littéraire (par le refus des notions d'ordre, d'harmonie, de vérité logocentrique) lui semblent refus du dogmatisme et ouverture à l'hétérogénéité dans la pensée et dạns la société.

Le lecteur, quant à lui, conserve ses doutes. Le sème "mode" est au cœur du terme "postmoderne" comme il est peut-être au centre du phénomène (voir Walter Moser que cite Janet Paterson, p. 3). Par ailleurs, le lecteur doit reconnaitre que la notion a une valeur heuristique; elle-est féconde puisqu'elle permet de mieux lire des textes particuliers et de percevoir des parentés d'œuvres dans une production qui, autrement, pourrait sembler cacophonique. Et il sait gré à l'auteure d'avoir introduit un peu d'ordre dans le discours sur une notion qui, précisément, refuse la possibilité d'un discours cohérent et qui, juste retour des choses, a attiré sa quote-part de logomachie. Ezra Pound proposait autrefois que l'on fusille les critiques qui se servent de notions théoriques de façon floue, inconstante et arbitraire. Avis aux intéressés: il me semble que l'exemple de Janet Paterson est à suivre par ceux qui voudraient éviter le poteau d'exécution.

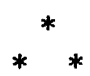

L'entreprise d'Anthony Purdy dans A Certain Difficulty of Being. Essays on the Quebec Novel ${ }^{2}$ est à la fois analogue à celle de Janet Paterson et foncièrement différente. Voici de nouveau un universitaire anglophone qui s'intéresse au roman québécois et qui se propose de l'étudier selon une approche théorique cohérente, dans ce cas, la narratologie. Cela dit; de profondes divergences séparent les deux livres (et au-delà de la question de savoir si tel roman, Kamouraska par exemple, est postmoderne ou non, voir Purdy, p. 131), car ils s'adressent à différents lecteurs. Janet Paterson s'adresse à un public francophone et on a par moments l'impression qu'elle veut inciter les Québécóis à prendre le train du postmodernisme: le Québec n'accuse-t-il pas un certain retard à reconnaître la fécondité de la notion? À peine peut-on dire que la notion est dans l'air du Québec ( p. 4) depuis 1985, alors que l'Amérique anglophone s'en sert depuis plus de vingt ans! Anthony Purdy, par ailleurs, écrit en anglais et s'adresse à ses compatriotes ${ }^{3}$ à qui il veut expliquer le roman québécois. Davantage, et c'est ici sans doute qu'Anthony Purdy se démarque le plus de Janet Paterson, sa position est celle d'un retrait et d'une distance critique à l'égard de la théorisation abusive des dernières années. Primauté des œuvres sur la méthode ou sur la théorie, tout en reconnaissant à celles-ci leur fécondité et leur rôle nourricier.

Anthony Purdy cherche à comprendre comment chacune des œuvres particulières fonctionne dans son individualité propre et non 
pas à les mesurer à l'aune d'une théorie ou à dégager leur indice d'exemplarité. Les œuvres étudiées; loin d'être choisies en fonction de leur adéquation à une poétique, et à plus forte raison à une poétique de la subversion des codes qui refuse radicalement toute possibilité de totalisation, le sont pour montrer comment chacune s'évertue à * faire du sens", à construire du sens avec des formes, dans un monde où le sens s'est effrité. C'est donc dire que son corpus n'est pas du tout celui de Janet Paterson. Les œuvres retenues sont d'avant 1970. Les préfaces des romans du XIX ${ }^{\mathrm{e}}$ siècle, Menaud, maître-draveur, Bonheur d'occasion, Poussière sur la ville retiennent son attention, et s'il étudie un roman d'Hubert Aquin (Prochain Épisode) ou Kamouraska d'Anne Hébert, c'est pour prendre le contre-pied d'une lecture postmoderne, pour insister sur la cohérence et l'intelligibilité des propos romanesques (avec ambivalence, toutefois, dans le cas de Prochain Épisode), et pour dégager le sens totalisé, une fois la dernière page du roman tournée. L'analyse de Kamouraska est d'ailleurs pour lui l'occasion de renvoyer dos à dos tenants du modernisme et du postmodernisme et de s'interroger sur ces querelles d'écoles (the debate surrounding postmodernism frequently has an air of depressing academic futility [p. 159, note 18]). En somme, si Anthony Purdy rejette la théorisation abusive et regrette que la "course à la théorie" soit souvent le recours à des béquilles qui dispensent de lire ou de vivre dans les textes, il est loin par ailleurs de méconnaître l'apport essentiel de la théorie et il ne veut certainement pas se priver de ses acquis. Disons plutôt que la "difficulté d'être" du titre est aussi celle de la critique qui, en ce début des années 1990, doit viser à être utile et ainsi sortir de sa tour d'ivoire savantasse.

Mais le titre, par le biais des épigraphes tirées d'Anne Hébert et d'Hubert Aquin (qui chacun renvoie à un autre, Saint-Denys Garneau et Fontenelle), est essentiellement rappel du mal-vivre québécois, de cette difficulté d'une collectivité d'être pleinement et de s'épanouir. Les œuvres qui témoignent des ambiguïtés d'un être-au-monde douloureux (en est-il d'autres?) sont celles qui retiennent l'attention de l'essayiste: I believe that the best and most interesting literature to come out of Quebec has always been structured by ontological uncertainty (p. xiii). Cette incertitude ontologique, cela va de soi, ne se déploie pas dans des œuvres sereines et d'une perfection olympienne. La difficulté d'être est aussi celle des œuvres qui peuvent difficilement arriver à leur plénitude et dont les ratés, les échecs, quelquefois flamboyants, assurent la réussite littéraire (si celle-ci se mesure par la fascination des lecteurs et le nombre de relectures, souvent irréconciliables). Problèmes de narration, fondamentalement: pourquoi les narrateurs de ces romans racontent-ils les histoires qu'ils racontent et pourquoi ne réussissent-ils pas toujours à faire ce qu'ils projetaient?

Le premier chapitre, en étudiant les préfaces des romans du XIX siècle, fait état de la première difficulté d'être, celle de la naissance elle- 
même. Le roman se nie avant de naître et semble ainsi venir au monde à son corps défendant. "Ceci n'est pas un roman" est l'énoncé fondamental et déroutant de ces préfaces. Il faut comprendre évidemment: voici un non-roman canadien (donc un livre sérieux), mais avec suffisamment d'éléments romanesques pour ne pas rebuter totalement le public formé au code culturel français; il n'y trouvera pas ce qui n'y est pas et ce à quoi il s'attend, mais peut-être y trouvera-t-il autre chose, plus valable, tout en n'étant pas complètement dérouté! Mauvaise conscience et justification tortueuse: cette naissance de mauvais augure trace un horizon littéraire qui ne sera jamais de tout repos.

Áinsi, l'étude de Menaud permettra de voir que le conflit entre métaphore et métonymie illustre l'ambivalence générique de l'œuvre (roman? poème? épopée?) et condamne le texte, tout autant que le protagoniste, à l'échec: L'analyse de Bonheur d'occasion nous montrera un écrivain sortant de son milieu familier pour aller à la découverte d'un monde autre (celui des prolétaires d'une métropole) et à la recherche, du même coup, d'une forme littéraire capable de traduire l'expérience de cet autre univers. La double quête sera partiellement ratée, du fait que l'auteur n'arrive pas à faire confiance ni au lecteur ni à la forme choisie. L'examen de Poussière sur la ville, de Prochain Épisode et de Kamouraska, œuvres d'une complexité narrative autre, plus moderne, entraîne des approches différentes. Le conflit entre le point de vue du médecin, du mari et du narrateur, tous le même personnage, produit des ambiguïtés insolubles dans l'œuvre de Langevin, et d'autant plus que le conflit des codes (code français d'une littérature sérieuse, "existentialiste", d'une part; code américain simplificateur d'une topographie de western, d'autre part) y est fondamental. Par ailleurs, Prochain Épisode sera étudié en relation avec le socio-texte et avec la pensée d'Aquin telle qu'elle se déploie dans ses essais, pour montrer que le roman est une entreprise de conscience, de désespoir libérateur. Kamouraska, pour sa part, nous fait passer de la sphère politique à la sphère privée, recherche d'un soi intime plutôt que d'un soi public, et Anthony Purdy insistera sur la cohérence et l'intelligibilité de sa structure narrative, dont la fragmentation n'est qu'apparente.

Toutes ces analyses sont substantielles et précises. Trop soucieuses peut-être de se situer face à telle ou telle autre lecture et de faire état de celles-ci. Mais il est vrai que les dettes sont nombreuses. Les lecteurs québécois trouveront donc dans ce livre de fréquents bilans du discours critique sur chacune des œuvres. J'ai surtout apprécié, quant à moi, l'étude sur Bonheur d'occasion qui fait une large place à un écrivain d'un autre univers culturel, Georges Orwell, qui avait à vivre les mêmes problèmes narratifs que Gabrielle Roy. C'est alors que le regard de l'autre, nourri de tout son bagage littéraire distinct du mien, m'intéresse le plus. 


\section{VOIX \& IMAGES / 48, printemps 1991}

1 Janet Paterson, Moments postmodernes dans le roman québécois, Ottawa, Presses de l'Université d'Ottawa, 1990, 126 p.

2 Anthony Purdy, A Certain Difficulty of Being. Esaays on the Quebec Novel, Montreal et Kingston, McGill-Queen's University Press, 1990, 176 p.

3. Du moins en théorie. Mais par ses références constantes à un univers culturel français (Sartre, Camus), des critiques et théoriciens francophones (français et québecois), et à une secondary literature (comme disent les Anglais) de langue française, il me semble évident qu'Anthony Purdy s'adresse autant à des lecteurs francophones. Ou à des lecteurs anglophones qui, par ailleurs, lisent le français et qui auraient pu lire son livre s'il avait été rédigé en français. On pourrait donc se demander à qui parle œ livre qui s'emploie à étudier comment on parle dans les romans québécois. Aux anglophones, certes (cela est bien évident dans un chapitre comme le troisieme qui fait des rapprochements fort judicieux entre les procédés narratifs de Georges Orwell et ceux de Gabrielle Roy); mais beaucoup plus souvent aux francophones, il me semble, malgré la langue utilisée. J'ajouterais, pour compliquer davantage la situation, qu'Anthony Purdy se defend bien d'avoir le regard d'un Canadien anglais sur la littérature québécoise. Britannique d'origine, il en serait venu a la littérature québécoise de par son intérêt pour les langues modernes. II ne partagerait pas le point de vue du Canada anglais, fatalement comparatiste et cherchant à canadianiser la littérature québecoise. Je ne suis pas en mesure de juger si cette prétention d'un regard autre de la part de quelqu'un qui vit au Canada anglais depuis 1973 est fondée. Mais je suis porté à croire que la position d'Anthony Purdy est la position traditionnelle de l'universitaire canadien-anglais: celui qui cherche à expliquer à ses compatriotes un aspect de la culture québécoise. 